\title{
Necessary Conditions for the Generic Global Rigidity of Frameworks on Surfaces
}

\author{
B. Jackson - T. A. McCourt • A. Nixon
}

Received: 10 June 2013 / Revised: 29 April 2014 / Accepted: 7 July 2014 /

Published online: 6 August 2014

(C) Springer Science+Business Media New York 2014

\begin{abstract}
A result due in its various parts to Hendrickson, Connelly, and Jackson and Jordán, provides a purely combinatorial characterisation of global rigidity for generic bar-joint frameworks in $\mathbb{R}^{2}$. The analogous conditions are known to be insufficient to characterise generic global rigidity in higher dimensions. Recently Laman-type characterisations of rigidity have been obtained for generic frameworks in $\mathbb{R}^{3}$ when the vertices are constrained to lie on various surfaces, such as the cylinder and the cone. In this paper we obtain analogues of Hendrickson's necessary conditions for the global rigidity of generic frameworks on the cylinder, cone and ellipsoid.
\end{abstract}

Keywords Rigidity - Global rigidity · Framework on a surface

Mathematics Subject Classification $52 \mathrm{C} 25 \cdot 05 \mathrm{C} 10 \cdot 53 \mathrm{~A} 05$

\section{Introduction}

A bar-joint framework in Euclidean space $\mathbb{R}^{d}$ is a geometric realisation of the vertices of a graph with the edges considered as (fixed length) bars between them. Such a framework is said to be rigid if there is no non-trivial continuous motion of the vertices

B. Jackson

School of Mathematical Sciences, Queen Mary University of London, London E1 4NS, UK

e-mail: b.jackson@qmul.ac.uk

T. A. McCourt · A. Nixon

Heilbronn Institute for Mathematical Research, School of Mathematics,

University of Bristol, Bristol BS8 1TW, UK

e-mail: tonynixon1@gmail.com

T. A. McCourt

e-mail: tom.a.mccourt@gmail.com 
in $\mathbb{R}^{d}$ which maintains bar-lengths, and is said to be flexible if it is not rigid. It is redundantly rigid if it remains rigid after deleting any single edge. A foundational theorem of Laman [11], obtained in 1970, asserts that the rigidity of a generically positioned framework in $\mathbb{R}^{2}$ depends only on the underlying graph and, furthermore, that these graphs are characterised in terms of a simple counting condition. Finding an analogous characterisation for the rigidity of generic frameworks in $\mathbb{R}^{3}$ is an important open problem.

Formally a framework $(G, p)$ in $\mathbb{R}^{d}$ is the combination of a finite graph $G=(V, E)$ and a map $p: V \rightarrow \mathbb{R}^{d}$. Two frameworks $(G, p)$ and $(G, q)$ are said to be equivalent if $\|p(v)-p(u)\|=\|q(v)-q(u)\|$ for all pairs of adjacent vertices $u, v \in V$. More strongly they are said to be congruent if $\|p(v)-p(u)\|=\|q(v)-q(u)\|$ holds for all pairs of vertices $u, v \in V$. A framework $(G, p)$ is globally rigid if every framework $(G, q)$ equivalent to $(G, p)$ is also congruent to $(G, p)$.

Hendrickson derived the following necessary conditions for generic global rigidity in $\mathbb{R}^{d}$.

Theorem 1 ([7]) Let $(G, p)$ be a generic globally rigid framework in $\mathbb{R}^{d}$. Then $G$ is a complete graph on at most $d+1$ vertices or $G$ is $(d+1)$-connected and $(G, p)$ is redundantly rigid in $\mathbb{R}^{d}$.

In the case of 2-dimensional frameworks these conditions are also sufficient.

Theorem 2 ([4,7] and [8]) Let $(G, p)$ be a generic framework in $\mathbb{R}^{2}$. Then $(G, p)$ is globally rigid if and only if either $G$ is a complete graph on at most three vertices or $G$ is 3-connected and $(G, p)$ is redundantly rigid.

Sufficiency follows by combining a geometric result due to Connelly and a combinatorial construction of Jackson and Jordán.

Both rigidity and global rigidity have far reaching applications. In particular, determining when a framework has a unique realisation up to congruence has applications in robotics [20] and in sensor networks [1].

Attention has recently been given to frameworks in $\mathbb{R}^{3}$ whose vertices are constrained to lie on 2-dimensional surfaces and analogues of Laman's theorem have been obtained for a variety of surfaces $[15,16]$. In this paper we consider the global rigidity of frameworks on these surfaces and obtain analogues of Hendrickson's necessary conditions for generic global rigidity. In the case when the surface is a sphere, Connelly and Whiteley [5] proved that a generic framework $(G, p)$ on the sphere is globally rigid if and only if a corresponding generic framework $(G, q)$ is globally rigid in the plane. We focus our attention on cylinders, cones and ellipsoids. We also include the sphere as it is covered by our proof technique and provides a complete proof that redundant rigidity is a necessary condition for generic global rigidity on the sphere, and hence also in the plane.

We conclude the introduction by giving a brief outline of the proof of our main result, that redundant rigidity is a necessary condition for generic global rigidity. We

\footnotetext{
${ }^{1}$ More precisely, Hendrickson proved the weaker result that almost all globally rigid frameworks in $\mathbb{R}^{d}$ are redundantly rigid. His proof technique can be extended to cover all generic frameworks using the theory of semi-algebraic sets and this extension has been taken to be implicit in the literature.
} 
adopt a similar approach to that of [7]. We consider the motion of the framework that results from deleting a non-redundant edge $e$ from a generic rigid framework $\left(G, p_{0}\right)$ on a surface $S$. We obtain a series of geometric results in Sects. 3-6 that enable us to show in Sect. 9 that this motion is diffeomorphic to a circle. We then use genericity to prove that the motion reaches a framework $\left(G-e, p_{1}\right)$ such that $\left(G, p_{1}\right)$ is equivalent but not congruent to $\left(G, p_{0}\right)$. We have to overcome two technical difficulties for surfaces which do not arise in [7]. We actually show in Sect. 9 that there is no isometry of $S$ which maps $\left(G, p_{0}\right)$ onto $\left(G, p_{1}\right)$. We prove that this apparently weaker conclusion implies that $\left(G, p_{0}\right)$ and $\left(G, p_{1}\right)$ are not congruent (as long as $G$ has enough vertices) in Sect. 7. In addition, to deduce that the motion of ( $\left.G-e, p_{0}\right)$ is diffeomorphic to a circle we need to show that it is bounded. This is straightforward for the sphere, cylinder and elipsoid, but requires a special argument for the cone. We give this in Sect. 8.

\section{Frameworks on Surfaces}

We assume henceforth that $G=(V, E)$ is a graph with $V=\left\{v_{1}, v_{2}, \ldots, v_{n}\right\}$ and $E=\left\{e_{1}, e_{2}, \ldots, e_{m}\right\}$. Let $S$ be a fixed surface in $\mathbb{R}^{3}$. A framework $(G, p)$ on $S$ is the combination of a finite graph $G=(V, E)$ and a map $p: V \rightarrow S$; such a framework is said to be rigid on $S$ if every continuous motion of the vertices on $S$ that preserves equivalence also preserves congruence; otherwise $(G, p)$ is said to be flexible on $S$. Moreover $(G, p)$ is: minimally rigid on $S$ if it is rigid on $S$ and, for every edge $e$ of $G$, the framework $(G-e, p)$ is flexible on $S$; redundantly rigid on $S$ if $(G-e, p)$ is rigid on $S$ for all $e \in E$; and globally rigid on $S$ if every framework $(G, q)$ on $S$ which is equivalent to $(G, p)$ is congruent to $(G, p)$.

An infinitesimal flex $s$ of $(G, p)$ on $S$ is a map $s: V \rightarrow \mathbb{R}^{3}$ such that $s(v)$ is tangential to $S$ for all $v \in V$ and $(p(u)-p(v)) \cdot(s(u)-s(v))=0$ for all $u v \in E$. The framework $(G, p)$ is infinitesimally rigid on $S$ if every infinitesimal flex of $(G, p)$ is an infinitesimal isometry of $S$.

In this paper we consider spheres, cylinders, cones and ellipsoids. These are natural examples of surfaces for which the dimension of the space of infinitesimal isometries is 3, 2, 1 and 0 , respectively. In fact our proof techniques apply to the following more general families of 'concentric' surfaces.

Let $r=\left(r_{1}, r_{2}, \ldots, r_{n}\right)$ be a vector of (not necessarily distinct) positive real numbers. For $1 \leq i \leq n$, let $\mathcal{S}_{i}=\left\{(x, y, z) \in \mathbb{R}^{3}: x^{2}+y^{2}+z^{2}=r_{i}\right\}$, $\mathcal{Y}_{i}=\left\{(x, y, z) \in \mathbb{R}^{3}: x^{2}+y^{2}=r_{i}\right\}, \mathcal{C}_{i}=\left\{(x, y, z) \in \mathbb{R}^{3}: x^{2}+y^{2}=r_{i} z^{2}\right\}$ and $\mathcal{E}_{i}=\left\{(x, y, z) \in \mathbb{R}^{3}: x^{2}+a y^{2}+b z^{2}=r_{i}\right\}$ for some fixed $a, b \in \mathbb{Q}$ with $1<a<b$. Let $\mathcal{S}=\bigcup_{i=1}^{n} \mathcal{S}_{i}, \mathcal{Y}=\bigcup_{i=1}^{n} \mathcal{Y}_{i}, \mathcal{C}=\bigcup_{i=1}^{n} \mathcal{C}_{i}$ and $\mathcal{E}=\bigcup_{i=1}^{n} \mathcal{E}_{i}$. We will use $S=\bigcup_{i=1}^{n} S_{i}$ to denote one of the four surfaces $(\mathcal{S}, \mathcal{Y}, \mathcal{C}, \mathcal{E})$ defined above and $\ell$ for the dimension of its space of infinitesimal isometries (so $\ell=3,2,1$ or 0 when $S=\mathcal{S}, \mathcal{Y}, \mathcal{C}$ or $\mathcal{E}$, respectively). We will occasionally use $S(r)$ when we wish to specify a particular vector $r$ and $S(1)$ for the special case when $r_{1}=r_{2}=\cdots=r_{n}$ (there is no loss in this case in assuming $r_{i}=1$ for all $1 \leq i \leq n$ ). We say that $(G, p)$ is a framework on $S$ if $p: V \rightarrow \mathbb{R}^{3}$ and $p\left(\bar{v}_{i}\right) \in S_{i}$ for all $1 \leq i \leq n$. 
The problem of determining whether or not a given framework on $S$ is rigid is a difficult problem in algebraic geometry. It becomes tractable however if we restrict our attention to 'generic' frameworks. We consider a framework $(G, p)$ on $S$ to be generic if $\operatorname{td}[\mathbb{Q}(r, p): \mathbb{Q}(r)]=2|V|$, where td $[\mathbb{Q}(r, p): \mathbb{Q}(r)]$ denotes the transcendence degree of the field extension. Thus $(G, p)$ is generic on $S$ if the coordinates of the vertices of $G$ are as algebraically independent as possible. For generic frameworks the problem of determining rigidity reduces to that of determining infinitesimal rigidity.

Theorem 3 ([15]) Let $(G, p)$ be a generic framework on $S$. Then $(G, p)$ is rigid on $S$ if and only if $G$ is a complete graph on at most 5 - $\ell$ vertices, or $(G, p)$ is infinitesimally rigid on $S$.

We note that [15] uses a different definition for a generic framework on $S$. Corollary 1 below verifies that any framework which satisfies our definition will also satisfy the definition given in [15].

Theorem 3, combined with Theorem 5 below, imply that the problem of determining generic rigidity on $S$ depends only on the underlying graph. This problem has been solved for three of our chosen surfaces.

Theorem 4 ([15,16]) Let $(G, p)$ be a generic framework on $S$ and suppose that $S \in$ $\{\mathcal{S}(r), \mathcal{Y}(r), \mathcal{C}(1)\}$. Then $(G, p)$ is minimally rigid on $S$ if and only if $G$ is $K_{n}$ for $1 \leq n \leq 5-\ell$ or $|E|=2|V|-\ell$ and every subgraph $H=\left(V^{\prime}, E^{\prime}\right)$ of $G$ has $\left|E^{\prime}\right| \leq 2\left|V^{\prime}\right|-\ell$.

It is an open problem to characterise generic rigidity on the ellipsoid. The analogous condition to that given in Theorem 4 is known to be necessary:

Lemma 1 ([15]) Let $(G, p)$ be a generic framework on $\mathcal{E}(r)$. If $(G, p)$ is minimally rigid then $G$ is $K_{n}$ for $1 \leq n \leq 4$ or $|E|=2|V|$ and every subgraph $H=\left(V^{\prime}, E^{\prime}\right)$ of $G$ has $\left|E^{\prime}\right| \leq 2\left|V^{\prime}\right|$.

However, the graph constructed by adding a vertex of degree two to $K_{5}$ has an infinitesimal flex for every generic realisation on the ellipsoid. This shows that the condition in Lemma 1 is not sufficient to imply generic rigidity.

\section{Generic Points and Smooth Manifolds}

Let $K, L$ be fields such that $\mathbb{Q} \subseteq K \subseteq L \subseteq \mathbb{C}$. Let $W$ be an algebraic variety over $K$ in $L^{n}$, i.e. $W=\left\{x \in L^{n}: f_{i}(x)=0\right.$ for all $\left.1 \leq i \leq m\right\}$ for some $f_{1}, f_{2}, \ldots, f_{m} \in$ $K[X]$. We assume that $W$ is irreducible; i.e. cannot be expressed as the union of two proper subvarieties. The dimension of $W, \operatorname{dim} W$, is the maximum length of a chain of subvarieties of $W$. A point $p \in W$ is generic over $K$ if every $h \in K[X]$ satisfying $h(p)=0$ has $h(x)=0$ for all $x \in W$. Given an integral domain $R$ we use $\operatorname{Fract}(R)$ to denote the field of fractions of $R$.

Lemma 2 Let $W$ be an irreducible variety over $K$ in $\mathbb{C}^{n}, p \in W$, and $I=\{f \in$ $K[X]: f(x)=0$ for all $x \in W\}$. Then: 
(a) $\operatorname{dim} W=\operatorname{td}[\operatorname{Fract}(K[X] / I): K]$;

(b) The map $h+I \mapsto h(p)$ is a surjective ring homomorphism from $K[X] / I$ to $K(p)$, and is a ring isomorphism if and only if $p$ is a generic point of $W$ over $K$;

(c) $\operatorname{td}[K(p): K] \leq \operatorname{dim} W$, and equality holds if and only if $p$ is a generic point of $W$ over $K$.

Proof Part (a) is well known (for example see [6, Exercise 3.20 (b)]) and Part (b) is elementary. Part (a) along with the first part of (b) implies that

$$
\operatorname{dim} W=\operatorname{td}[\operatorname{Fract}(K[X] / I): K] \geq \operatorname{td}[K(p): K] .
$$

The second part of (b) tells us that if $p$ is generic then equality holds in the above inequality. It remains to show that $p$ is a generic point of $W$ over $K$ when $\operatorname{td}[K(p)$ : $K]=\operatorname{dim} W$. Let $h \in K[X]$ with $h(p)=0, W_{1}=\{x \in W: h(x)=0\}$, and let $W_{2}$ be the irreducible component of $W_{1}$ which contains $p$. The argument in the second sentence of the proof tells us that $\operatorname{dim} W_{2} \geq \operatorname{td}[K(p): K]=\operatorname{dim} W$. The definition of dimension and the fact that $W_{2}$ is a subvariety of $W$ now imply that $W_{2}=W$. Hence $h(x)=0$ for all $x \in W$ and $p$ is a generic point of $W$ over $K$.

Corollary 1 Let $K$ be a field with $\mathbb{Q} \subseteq K \subseteq \mathbb{R}, W$ be an irreducible variety over $K$ in $\mathbb{C}^{n}$ and $p \in W \cap \mathbb{R}^{n}$. If $\operatorname{td}[K(p): K]=\operatorname{dim} W$ then $p$ is a generic point of $W$ and hence is also a generic point of $W \cap \mathbb{R}^{n}$.

Let $X$ be a smooth manifold and $f: X \rightarrow \mathbb{R}^{m}$ be a smooth map. Then $x \in X$ is said to be a regular point of $f$ if $\left.d f\right|_{x}$ has maximum rank and is a critical point of $f$ otherwise. Also $f(x)$ is said to be a regular value of $f$ if, for all $y \in f^{-1}(f(x)), y$ is a regular point of $f$; otherwise $f(x)$ is a critical value of $f$.

Lemma 3 ([9]) Let $M$ be a smooth manifold and $x \in M$. Suppose $\theta: M \rightarrow \mathbb{R}^{a}$ and $F: M \rightarrow \mathbb{R}^{b}$ are smooth maps. Define $H: M \rightarrow \mathbb{R}^{a+b}$ by $H(y)=(F(y), \theta(y))$. Suppose $\theta(x)$ is a regular value of $\theta$. Let $X$ be the submanifold $\theta^{-1}(\theta(x))$ of $M$, and let $f$ be the restriction of $F$ to $X$. Then $\left.\operatorname{rank} d f\right|_{x}=\left.\operatorname{rank} d H\right|_{x}-\left.\operatorname{rank} d \theta\right|_{x}$.

Note that $\theta^{-1}(\theta(x))$ is a submanifold of $M$ by the following well known result, see for example [13, p. 11, Lemma 1].

Lemma 4 Let $X$ and $Y$ be smooth manifolds and $f: X \rightarrow Y$ be a smooth map. Suppose that $S$ has dimension $m, x \in X, f(x)$ is a regular value of $f$ and $\left.\operatorname{rank} d f\right|_{x}=$ $t$. Then $f^{-1}(f(x))$ is an $(m-t)$-dimensional smooth manifold.

\section{The Rigidity Map}

Given the graph $G$, the rigidity map $f_{G}: \mathbb{R}^{3 n} \rightarrow \mathbb{R}^{m}$ is defined by $f_{G}(p)=$ $\left(\left\|e_{1}\right\|^{2}, \ldots,\left\|e_{m}\right\|^{2}\right)$ where $\left\|e_{i}\right\|^{2}=\left\|p\left(v_{j}\right)-p\left(v_{k}\right)\right\|^{2}$ when $e_{i}=v_{j} v_{k}$. Given the surface $S$, the $S$-rigidity map $F_{G}: \mathbb{R}^{3 n} \rightarrow \mathbb{R}^{m+n}$ is defined by $F_{G}=\left(f_{G}, \theta_{G}\right)$ where $\theta_{G}: \mathbb{R}^{3 n} \rightarrow \mathbb{R}^{n}$ is given by $\theta_{G}(p)=\left(h\left(p\left(v_{1}\right)\right), \ldots, h\left(p\left(v_{n}\right)\right)\right)$ and: 


$$
h\left(x_{i}, y_{i}, z_{i}\right)= \begin{cases}x_{i}^{2}+y_{i}^{2}+z_{i}^{2}-r_{i} & \text { if } S=\mathcal{S}, \\ x_{i}^{2}+y_{i}^{2}-r_{i} & \text { if } S=\mathcal{Y}, \\ x_{i}^{2}+y_{i}^{2}-r_{i} z_{i}^{2} & \text { if } S=\mathcal{C}, \\ x_{i}^{2}+a y_{i}^{2}+b z_{i}^{2}-r_{i} & \text { if } S=\mathcal{E} .\end{cases}
$$

We will consider the restriction of $F_{G}$ to the irreducible algebraic variety $\mathcal{W}=$ $S_{1} \times S_{2} \times \cdots \times S_{n}$. The Jacobian matrix for the derivative of $F_{G}$ evaluated at any point $p \in \mathcal{W}$ is (up to scaling) the rigidity matrix for the framework $(G, p)$ on $S$. It is shown in [15] that the null space of this matrix is the space of infinitesimal flexes of $(G, p)$ on $S$. This allows us to characterise infinitesimally rigid frameworks in terms of $d F_{G}$.

Theorem 5 ([15]) Let $(G, p)$ be a framework on $S$. Then $(G, p)$ is infinitesimally rigid if and only if rank $\left.d F_{G}\right|_{p}=3 n-\ell$.

Note that for any $p=\left(x_{1}, y_{1}, z_{1}, \ldots, x_{n}, y_{n}, z_{n}\right) \in \mathcal{W}$ we have

$$
\left.\mathrm{d} \theta_{G}\right|_{p}=\left[\begin{array}{cccc}
d h\left(p\left(v_{1}\right)\right) & 0 & \ldots & 0 \\
0 & d h\left(p\left(v_{2}\right)\right) & \ldots & 0 \\
\vdots & & \ddots & \vdots \\
0 & 0 & \ldots & d h\left(p\left(v_{n}\right)\right)
\end{array}\right],
$$

where

$$
d h\left(x_{i}, y_{i}, z_{i}\right)= \begin{cases}2\left(x_{i}, y_{i}, z_{i}\right) & \text { if } S=\mathcal{S}, \\ 2\left(x_{i}, y_{i}, 0\right) & \text { if } S=\mathcal{Y}, \\ 2\left(x_{i}, y_{i},-r_{i} z_{i}\right) & \text { if } S=\mathcal{C}, \\ 2\left(x_{i}, a y_{i}, b z_{i}\right) & \text { if } S=\mathcal{E} .\end{cases}
$$

It follows that rank $\left.\mathrm{d} \theta_{G}\right|_{p}=n$ unless $p\left(v_{i}\right)=(0,0,0)$ for some $1 \leq i \leq n$ (which can only occur if $S=\mathcal{C}$ ). Thus $p$ is a regular point of $\theta_{G}$ for all such $p$. Moreover we have $\theta_{G}(p)=0$, and 0 is a regular value of $\theta_{G}$ for all $p \in \mathcal{W}$ when $S \in\{\mathcal{S}, \mathcal{Y}, \mathcal{E}\}$. (Lemma 4 applied to $\theta_{G}$ at any $p \in \mathcal{W}$ now confirms that $\mathcal{W}$ is a manifold of dimension $3 n-n=2 n$ in these three cases.)

We say that two frameworks $(G, p)$ and $(G, q)$ on $S$ are $S$-congruent if there is an isometry of $S$ which maps $(G, p)$ to $(G, q)$. Note that $S$-congruence is a stronger condition than congruence. For example, we may have two equivalent realisations of $K_{3}$ on the cylinder which are not $\mathcal{Y}$-congruent (but clearly must be congruent). The framework $(G, p)$ is in standard position on $S$ (with respect to the fixed ordering of the vertices) if $p\left(v_{1}\right)=\left(x_{1}, y_{1}, z_{1}\right), p\left(v_{2}\right)=\left(x_{2}, y_{2}, z_{2}\right)$ and: $\left(x_{1}, y_{1}, z_{1}\right)=\left(0, y_{1}, 0\right)$ and $x_{2}=0$ if $S=\mathcal{S} ;\left(x_{1}, y_{1}, z_{1}\right)=\left(0, y_{1}, 0\right)$ if $S=\mathcal{Y}$; and $x_{1}=0$ if $S=\mathcal{C}$. When $S=\mathcal{E}$ all frameworks are considered to be in standard position. It is easy to see that any framework $(G, p)$ on $S$ is $S$-congruent to a framework in standard position on $S$. We consider frameworks $(G, p)$ in standard position in order to factor out the continuous isometries of $S$. 
We will need a version of the $S$-rigidity map for frameworks which are constrained to lie in standard position on $S$. Define $\alpha: \mathbb{R}^{3 n} \rightarrow \mathbb{R}^{\ell}$ by

$$
\alpha\left(x_{1}, y_{1}, z_{1}, \ldots, x_{n}, y_{n}, z_{n}\right)= \begin{cases}\left(x_{1}, z_{1}, x_{2}\right) & \text { if } S=\mathcal{S} \\ \left(x_{1}, z_{1}\right) & \text { if } S=\mathcal{Y} \\ x_{1} & \text { if } S=\mathcal{C}\end{cases}
$$

Define $\theta_{G}^{*}: \mathbb{R}^{3 n} \rightarrow \mathbb{R}^{n+\ell}$ by $\theta_{G}^{*}=\left(\theta_{G}, \alpha\right)$ if $S \in\{\mathcal{S}, \mathcal{Y}, \mathcal{C}\}$ and $\theta_{G}^{*}=\theta_{G}$ if $S=\mathcal{E}$. Let $F_{G}^{*}: \mathbb{R}^{3 n} \rightarrow \mathbb{R}^{m+n+\ell}$ by $F_{G}^{*}=\left(f_{G}, \theta_{G}^{*}\right)$. Then the null space of $\left.d F_{G}^{*}\right|_{p}$ is the space of all infinitesimal flexes of $(G, p)$ which leave the coordinates in $\alpha(p)$ fixed.

We say a framework $(G, p)$ on $S$ is independent if rank $\left.d F_{G}\right|_{p}=m+n$ i.e. the rows of (the Jacobian matrix for) $\left.d F_{G}\right|_{p}$ are linearly independent.

Lemma 5 Let $(G, p)$ be an independent framework on $S$. Then $\left.d F_{G}^{*}\right|_{p}$ has linearly independent rows and hence rank $\left.d F_{G}^{*}\right|_{p}=m+n+\ell$.

Proof We first consider the case when $(G, p)$ is minimally rigid, and hence infinitesimally rigid. Since the null space of $\left.d F_{G}^{*}\right|_{p}$ is the space of all infinitesimal flexes of $(G, p)$ which leave the coordinates in $\alpha(p)$ fixed, this space is trivial. Hence rank $\left.d F_{G}^{*}\right|_{p}=3 n=n+m+\ell$ and the rows of $\left.d F_{G}^{*}\right|_{p}$ are linearly independent. The case when $(G, p)$ is not minimally rigid follows since any independent framework $(G, p)$ can be extended to a minimally rigid framework $(H, p)$, and $\left.d F_{G}^{*}\right|_{p}$ can then be obtained by deleting rows from $\left.d F_{H}^{*}\right|_{p}$.

We next use Lemma 5 to obtain an analogous result for the restriction of the rigidity map $f_{G}$ to the $(2 n-\ell)$-dimensional manifold

$\mathcal{M}=\{p \in \mathcal{W}:(G, p)$ is in standard position on $S$ and $p(v) \neq(0,0,0)$ for all $v \in V\}$.

(The condition that $p(v) \neq(0,0,0)$ for all $v \in V$ ensures that $\mathcal{M}$ is a manifold. It is only relevant when $\mathcal{W}=\mathcal{C}$.) Let $f_{[G]}=\left.f_{G}\right|_{\mathcal{M}}$.

Lemma 6 Let $(G, p)$ be an independent framework in standard position on $S$. Then $\left.\operatorname{rank} d f_{[G]}\right|_{p}=m$ and hence $p$ is a regular point of $f_{[G]}$.

Proof We first consider the case when $S \in\{\mathcal{S}, \mathcal{Y}, \mathcal{E}\}$. We can use a similar argument to that given after Theorem 5 to show that rank $\left.d \theta_{G}^{*}\right|_{q}=n+\ell$ for all $q \in \mathcal{W}$. Since $q \in \mathcal{W}$ whenever $\theta_{G}^{*}(q)=\theta_{G}^{*}(p)$, this implies that $\theta_{G}^{*}(p)$ is a regular value of $\theta_{G}^{*}$. Since $\mathcal{M}=\left(\theta_{G}^{*}\right)^{-1}\left(\theta_{G}^{*}(p)\right)$, we may now use Lemmas 3 and 5 to deduce that

$$
\left.\operatorname{rank} d f_{[G]}\right|_{p}=\left.\operatorname{rank} d F_{G}^{*}\right|_{p}-\left.\operatorname{rank} d \theta_{G}^{*}\right|_{p}=m+n+\ell-(n+\ell)=m .
$$

We proceed similarly in the case when $S=\mathcal{C}$, but we have to restrict the domain of $F_{G}^{*}$ to an open neighbourhood of $p$ which contains no critical points of $F_{G}^{*}$, i.e. contains no points $p$ with $p(v)=(0,0,0)$ for some $v \in V$, otherwise $p$ may no longer be a regular value of $\theta_{G}^{*}$. 


\section{Quasi-generic Frameworks}

A framework $(G, p)$ is quasi-generic on $S$ if it is $S$-congruent to a generic framework. ${ }^{2}$

Lemma 7 Let $(G, p)$ be a minimally rigid quasi-generic framework on $S$. Then

$$
\operatorname{td}\left[\mathbb{Q}\left(r, f_{G}(p)\right): \mathbb{Q}(r)\right]=m .
$$

Proof Without loss of generality we may assume $(G, p)$ is in standard position. Let $f_{[G]}(p)=\left(\beta_{1}, \ldots, \beta_{m}\right)=\beta$. Suppose $g(\beta)=0$ for some polynomial $g$ with coefficients in $\mathbb{Q}(r)$. Then $g \circ f_{[G]}(p)=0$. Since $(G, p)$ is quasi-generic and $g \circ f_{[G]}$ is a polynomial with coefficients in $\mathbb{Q}(r)$, Corollary 1 implies that $g \circ f_{[G]}(q)=0$ for all $q \in \mathcal{W}$. In particular $g \circ f_{[G]}(q)=0$ for all $q \in \mathcal{M}$. By Lemma 6, rank $\left.\mathrm{d} f_{[G]}\right|_{q}=m$. Since $(G, p)$ is minimally rigid, $\mathcal{M}$ is $m$-dimensional and hence the Inverse Function Theorem implies that $f_{[G]}$ maps some open neighbourhood $U$ of $p$ in $\mathcal{M}$ diffeomorphically onto some neighbourhood $f_{[G]}(U)$ of $f_{[G]}(p)$ in $\mathbb{R}^{m}$. Then $g(y)=0$ for all $y$ in the open subset $f_{[G]}(U)$ of $\mathbb{R}^{m}$. This implies that $g$ must be the zero polynomial and hence $\left\{\beta_{1}, \ldots, \beta_{m}\right\}$ is algebraically independent over $\mathbb{Q}(r)$.

Lemma 8 A framework $(G, p)$ on $S$ is quasi-generic if and only if $(G, p)$ is $S$ congruent to a framework $(G, q)$ in standard position with $\operatorname{td}[\mathbb{Q}(r, q): \mathbb{Q}(r)]=$ $2 n-\ell$.

Proof Suppose that $(G, p)$ is quasi-generic. Then $(G, p)$ is $S$-congruent to a framework $(G, q)$ in standard position. Since the definition of quasi-generic depends only on $p$, we may assume, without loss of generality, that $G$ is minimally rigid. By Lemma $7, \operatorname{td}\left[\mathbb{Q}\left(r, f_{G}(p)\right): \mathbb{Q}(r)\right]=\operatorname{td}\left[\mathbb{Q}\left(r, f_{G}(q)\right): \mathbb{Q}(r)\right]=2 n-\ell$. Let $K$ and $L$ be the algebraic closures of $\mathbb{Q}\left(r, f_{G}(q)\right)$ and $\mathbb{Q}(r, q)$ respectively. Since $K \subseteq L$ we have

$$
\operatorname{td}[\mathbb{Q}(r, q): \mathbb{Q}(r)]=\operatorname{td}[L: \mathbb{Q}(r)] \geq \operatorname{td}[K: \mathbb{Q}(r)]=2 n-\ell
$$

Since $(G, q)$ is in standard position on $S$, we also have td $[\mathbb{Q}(r, q): \mathbb{Q}(r)] \leq 2 n-\ell$. Hence td $[\mathbb{Q}(r, q): \mathbb{Q}(r)]=2 n-\ell$.

Now suppose $(G, p)$ is $S$-congruent to a framework $(G, q)$ in standard position with $\operatorname{td}[\mathbb{Q}(r, q): \mathbb{Q}(r)]=2 n-\ell$. Let $q=\left(q\left(v_{1}\right), x_{2}, y_{2}, z_{2}, \ldots, x_{n}, y_{n}, z_{n}\right)$. When $S=\mathcal{E}$, we have $\ell=0$ and $(G, q)$ is generic so we are done.

Suppose $S=\mathcal{Y}$. As $\operatorname{td}[\mathbb{R}: \mathbb{Q}(r)]=\infty$ we may choose $\theta$ such that $\left\{\sin \theta, x_{2}, \ldots, x_{n}, z_{2}, \ldots, z_{n}\right\}$ is algebraically independent over $\mathbb{Q}(r)$. Apply the rotation

$$
\left[\begin{array}{ccc}
\cos \theta & -\sin \theta & 0 \\
\sin \theta & \cos \theta & 0 \\
0 & 0 & 1
\end{array}\right]
$$

\footnotetext{
2 It is convenient for our purposes to regard quasi-genericness as a property of a framework $(G, p)$ even though its definition depends only on the configuration $p$.
} 
to each of the vertices of $(G, q)$ to achieve the equivalent framework $\left(G, q^{\prime}\right)$. Then

$$
\begin{aligned}
q^{\prime}= & \left(-y_{1} \sin \theta, y_{1} \cos \theta, 0, x_{2} \cos \theta-y_{2} \sin \theta, x_{2} \sin \theta+y_{2} \cos \theta, z_{2}, x_{3} \cos \theta\right. \\
& -y_{3} \sin \theta, x_{3} \sin \theta+y_{3} \cos \theta, z_{3}, \ldots, x_{n} \cos \theta-y_{n} \sin \theta, x_{n} \sin \theta \\
& \left.+y_{n} \cos \theta, z_{n}\right) .
\end{aligned}
$$

As $\left\{\sin \theta, x_{2}, \ldots, x_{n}, z_{2}, \ldots, z_{n}\right\}$ is algebraically independent over $\mathbb{Q}(r), S=\mathcal{Y}$ and $x_{i}^{2}+y_{i}^{2}=r_{i}$ for $2 \leq i \leq n, \quad\left\{\sin \theta, x_{2} \cos \theta-y_{2} \sin \theta, \ldots, x_{n} \cos \theta-\right.$ $\left.y_{n} \sin \theta, z_{1}, \ldots, z_{n}\right\}$ is algebraically independent over $\mathbb{Q}(r)$. Next choose $t \in \mathbb{R}$ such that $T=\left\{\sin \theta, x_{2} \cos \theta-y_{2} \sin \theta, \ldots, x_{n} \cos \theta-y_{n} \sin \theta, z_{1}+t, \ldots, z_{n}+t\right\}$ is algebraically independent over $\mathbb{Q}(r)$. Translating $\left(G, q^{\prime}\right)$ parallel to the $z$-axis by $t$ we reach the equivalent framework $\left(G, q^{\prime \prime}\right)$ where $q^{\prime \prime}=\left(-y_{1} \sin \theta, y_{1} \cos \theta, t, x_{2} \cos \theta-\right.$ $\left.y_{2} \sin \theta, x_{2} \sin \theta+y_{2} \cos \theta, z_{2}+t, \ldots, x_{n} \cos \theta-y_{n} \sin \theta, x_{n} \sin \theta+y_{n} \cos \theta, z_{n}+t\right)$. As $T$ is algebraically independent over $\mathbb{Q}(r)$ we have that $\operatorname{td}\left[\mathbb{Q}\left(r, q^{\prime \prime}\right): \mathbb{Q}(r)\right]=2 n$. Thus $q^{\prime \prime}$ is generic on $\mathcal{Y}$. Since $(G, p)$ is $S$-congruent to $\left(G, q^{\prime \prime}\right),(G, p)$ is quasigeneric.

The remaining cases, when $S=\mathcal{S}$ or $S=\mathcal{C}$, follow by a similar argument.

Lemma 9 Let $(G, p)$ be a minimally rigid framework on $S$. Then $(G, p)$ is quasigeneric if and only if $\operatorname{td}\left[\mathbb{Q}\left(r, f_{G}(p)\right): \mathbb{Q}(r)\right]=2 n-\ell$.

Proof We may assume that $(G, p)$ is in standard position on $S$. If $(G, p)$ is quasigeneric, then $\operatorname{td}\left[\mathbb{Q}\left(r, f_{G}(p)\right): \mathbb{Q}(r)\right]=2 n-\ell$ by Lemma 7 .

Suppose on the other hand that $\operatorname{td}\left[\mathbb{Q}\left(r, f_{G}(p)\right): \mathbb{Q}(r)\right]=2 n-\ell$. Since $(G, p)$ is in standard position on $S$ we have td $[\mathbb{Q}(r, p): \mathbb{Q}(r)] \leq 2 n-\ell$. Since $\mathbb{Q}\left(r, f_{G}(p)\right) \subseteq$ $\mathbb{Q}(r, p)$ and $\operatorname{td}\left[\mathbb{Q}\left(r, f_{G}(p)\right): \mathbb{Q}(r)\right]=2 n-\ell$, we must have td $[\mathbb{Q}(r, p): \mathbb{Q}(r)]=$ $2 n-\ell$. Lemma 8 now tells us that $(G, p)$ is quasi-generic.

\section{Regular Maps}

Suppose $(G, p)$ is in standard position on $S$. Lemma 6 implies that $p$ is a regular point of $f_{[G]}$ when $(G, p)$ is independent. We will use the following fundamental theorems to prove that $f_{[G]}(p)$ is a regular value of $f_{[G]}$ when $(G, p)$ is quasi-generic.

Let $\mathbb{K}$ be a field such that $\mathbb{Q} \subseteq \mathbb{K} \subseteq \mathbb{R}$. Recall that a subset $A$ of $\mathbb{R}^{n}$ is semi-algebraic over $\mathbb{K}$ if it can be expressed as a finite union of sets of the form

$$
\left\{x \in \mathbb{R}^{n}: P_{i}(x)=0 \text { for } 1 \leq i \leq s \text { and } Q_{j}(x)>0 \text { for } 1 \leq j \leq t\right\},
$$

where $P_{i} \in \mathbb{K}\left[X_{1}, \ldots, X_{n}\right]$ for $1 \leq i \leq s$, and $Q_{j} \in \mathbb{K}\left[X_{1}, \ldots, X_{n}\right]$ for $1 \leq j \leq t$.

Theorem 6 (Tarski-Seidenberg $[17,19]$ ) Let $A \subset \mathbb{R}^{n+k}$ be semi-algebraic over $\mathbb{K}$ and $\pi: \mathbb{R}^{n+k} \rightarrow \mathbb{R}^{n}$ be the projection onto the first $n$ coordinates. Then $\pi(A)$ is semi-algebraic over $\mathbb{K}$.

Theorem 7 (Sard, see [13, p. 16]) Let $f: U \rightarrow \mathbb{R}^{n}$ be a smooth map defined on an open subset $U$ of $\mathbb{R}^{m}$ and $C$ be the set of critical points of $f$. Then $f(C)$ has Lebesque measure zero in $\mathbb{R}^{n}$. 
We also need the following elementary result, see for example [9, Lemma 3.3].

Lemma 10 Let $M$ be a smooth manifold and let $f: M \rightarrow \mathbb{R}^{n}$ be a smooth map. Let $x \in M$ and choose an open neighbourhood $U$ of $x$ on $M$ such that $U$ is diffeomorphic to $\mathbb{R}^{m}$. Let $g$ be the restriction of $f$ to $U$ and let $x$ be a regular point of $g$. Suppose that the rank of $\left.d g\right|_{x}$ is $n$. Then there exists an open neighbourhood $W \subseteq U$ of $x$ such that $g(W)$ is an open neighbourhood of $g(x)$ in $\mathbb{R}^{n}$.

Lemma 11 Let $\left(G, p_{0}\right)$ be a quasi-generic framework in standard position on $S$. Then $f_{[G]}\left(p_{0}\right)$ is a regular value of $f_{[G]}$.

Proof Let $\left(G, p_{0}\right)$ be congruent to the generic framework $\left(G, p_{1}\right)$. We argue for $\left(G, p_{1}\right)$ and first consider the case when $\left(G, p_{1}\right)$ is independent. Let $t=$ $\max \left\{\left.\operatorname{rank} d f_{[G]}\right|_{p}: p \in \mathcal{M}\right\}$. Let

$$
A=\left\{(p, q) \in \mathcal{M} \times \mathcal{M}: f_{[G]}(p)=f_{[G]}(q) \text { and }\left.\operatorname{rank} d f_{[G]}\right|_{q}<t\right\}
$$

and

$$
A^{\prime}=\{p \in \mathcal{M}: \text { there exists } q \in \mathcal{M} \text { such that }(p, q) \in A\}
$$

The set $A$ is semi-algebraic over $\mathbb{K}=\mathbb{Q}(r)$, so Theorem 6 implies that $A^{\prime}$ is semialgebraic over $\mathbb{K}$.

Suppose that $p_{1} \in A^{\prime}$. Then there exists a set

$$
A^{\prime \prime}=\left\{x \in \mathcal{M}: P_{i}(x)=0 \text { and } Q_{j}(x)>0 \text { for } 1 \leq i \leq c \text { and } 1 \leq j \leq d\right\} \subseteq A^{\prime}
$$

that contains $p_{1}$ where $P_{i}, Q_{j} \in \mathbb{K}\left[x_{1}, y_{1}, z_{1}, x_{2}, \ldots, z_{n}\right]$ and $c, d \geq 0$. Let $I=\{g \in$ $\mathbb{K}\left[x_{1}, y_{1}, z_{1}, x_{2}, \ldots, z_{n}\right]: g(x)=0$ for all $\left.x \in \mathcal{W}\right\}$. Since $p_{1}$ is generic on $\mathcal{W}$ and $P_{i}\left(p_{1}\right)=0$, we have $P_{i} \in I$ for all $1 \leq i \leq c$. Hence

$$
A^{\prime \prime}=\left\{x \in \mathcal{M}: Q_{j}(x)>0 \text { for } 1 \leq j \leq d\right\} .
$$

This implies that there exists a neighbourhood $W$ of $p_{1}$ on $\mathcal{M}$ such that $W \subseteq A^{\prime \prime}$. By Lemmas 6 and 10 we can choose $W^{\prime} \subset W$ such that $f_{[G]}\left(W^{\prime}\right)$ is an open subset of $\mathbb{R}^{m}$. Then each point of $f_{[G]}\left(W^{\prime}\right)$ is a critical value of $f_{[G]}$, contradicting Theorem 7. Thus $p_{1} \notin A^{\prime}$. Since we also have rank $\left.d f_{[G]}\right|_{p_{1}}=t$ by Lemma $6, f_{[G]}\left(p_{1}\right)$ is a regular value of $f_{[G]}$.

When $\left(G, p_{1}\right)$ is not independent we choose a maximal subgraph $H$ such that $\left(H, p_{1}\right)$ is independent. Then $f_{[H]}\left(p_{1}\right)$ is a regular value of $f_{[H]}$ and hence $f_{[G]}\left(p_{1}\right)$ is a regular value of $f_{[G]}$. Since $f_{[G]}\left(p_{1}\right)$ is a regular value of $f_{[G]}$ and $\left(G, p_{0}\right)$ is congruent to $\left(G, p_{1}\right)$ it follows that $f_{[G]}\left(p_{0}\right)$ is a regular value of $f_{[G]}$.

Lemma 12 Let $(G, p)$ be a quasi-generic framework in standard position on $S$ and $r=\operatorname{rank} d f_{[G]}(p)$. Then $f_{[G]}^{-1}\left(f_{[G]}(p)\right)$ is a $(2 n-\ell-r)$-dimensional smooth manifold.

Proof The domain of $f_{[G]}$ is $\mathcal{M}$ which is a $(2 n-\ell)$-dimensional manifold.

Lemma 11 shows that $f_{[G]}(p)$ is a regular value of $f_{[G]}$. The result now follows by applying Lemma 4. 


\section{Unique Surfaces Containing a Given Set of Points}

We show in this section that if $G$ has sufficiently many vertices and $(G, p)$ and $(G, q)$ are congruent frameworks on $S$, then they are $S$-congruent; i.e. there is an isometry of $S$ which maps $(G, p)$ onto $(G, q)$.

We say that two surfaces in $\mathbb{R}^{3}$ are congruent if there is an isometry of $\mathbb{R}^{3}$ mapping one to the other. We first show that if we choose a set $V$ of sufficiently many generic points on $S$, then the only surface which is congruent to $S$ and contains $V$ is $S$ itself.

Lemma 13 Suppose $\left(K_{7-\ell}, p\right)$ is a generic realisation of $K_{7-\ell}$ on $S$. Let $T$ be another surface in $\mathbb{R}^{3}$ which is congruent to $S$ and contains $\left(K_{7-\ell}, p\right)$. Then $T=S$.

Proof We first consider the case when $S=\mathcal{Y}$. Then $\ell=2$, and $T=\bigcup_{i=1}^{5} T_{i}$ is a congruent family of concentric cylinders containing $\left(K_{5}, p\right)$. We may characterise $T$ by four parameters $c_{1}, c_{2}, c_{3}, c_{4}$. For example, when the axis of $T$ has a non-zero $z$-component, we can take $\left(c_{1}, c_{2}, 1\right)$ to be a direction vector for this axis and $\left(c_{3}, c_{4}, 0\right)$ to be the point where it crosses the $x y$-plane. Let $\hat{\mathcal{Y}}_{i}, \hat{T}_{i}$ be the complex extensions of $\mathcal{Y}_{i}$ and $T_{i}$, respectively, i.e. the sets of all complex solutions to the equations which define $\mathcal{Y}_{i}$ and $T_{i}$. Let $\hat{Z}_{i}$ be the irreducible component of $\hat{\mathcal{Y}}_{i} \cap \hat{T}_{i}$ which contains $p\left(v_{i}\right)$ for all $1 \leq i \leq 5$. Each $Z_{i}$ is a proper subvariety of $\hat{\mathcal{Y}}_{i}$ so has dimension at most 1. Let $Z$ be the direct product of $Z_{1}, \ldots, Z_{5}$. Then $Z$ is irreducible and $\operatorname{dim} Z \leq 5$. Lemma 2(c) now implies that $\operatorname{td}[\mathbb{K}(p): \mathbb{K}] \leq 5$, where $\mathbb{K}=\mathbb{Q}\left(r, c_{1}, c_{2}, c_{3}, c_{4}\right)$. Thus $\operatorname{td}[\mathbb{K}(p): \mathbb{Q}(r)] \leq 9$. However, since $p$ is generic on $\mathcal{W}$ and $\operatorname{dim} \mathcal{W}=10$, Lemma 2(c) also gives td $[\mathbb{Q}(r, p): \mathbb{Q}(r)]=10$. This is a contradiction since $\mathbb{Q}(r, p) \subseteq \mathbb{K}(p)$.

The other cases follow similarly, using the fact that $T$ can be characterised by a set of $6-\ell$ parameters.

Lemma 14 Suppose $\left(K_{n}, p\right)$ is a generic framework on $S$ and $n \geq 7-\ell$. Let $\left(K_{n}, q\right)$ be a framework on $S$ equivalent to $\left(K_{n}, p\right)$. Then there is an isometry $\iota$ of $S$ such that $\iota(p)=q$.

Proof It suffices to prove the case $n=7-\ell$. Since $\left(K_{7-\ell}, p\right)$ and $\left(K_{7-\ell}, q\right)$ are congruent there is an isometry $\iota$ of $\mathbb{R}^{3}$ which maps $\left(K_{7-\ell}, p\right)$ onto $\left(K_{7-\ell}, q\right)$. Then $\iota(S)$ is a surface in $\mathbb{R}^{3}$ which is congruent to $S$. By Lemma 13, $S$ is the unique such surface containing $\left(K_{7-\ell}, q\right)$. Hence $\iota(S)=S$ and $\iota$ is an isometry of $S$.

Note that, in the case when $S=\mathcal{E}$, there are no continuous isometries so $\iota$ must be a discrete isometry.

\section{Compactness}

Given a framework $(G, p)$ on $S$, we define the configuration space $C(G, p)$ by $C(G, p)=\left\{q \in \mathbb{R}^{3}:(G, q)\right.$ is in standard position on $S$ and equivalent to $\left.(G, p)\right\}$.

We will show in the next section that if $(G, p)$ is rigid and generic, and $(G-e, p)$ is not rigid for some $e \in E$, then $(G, p)$ is not globally rigid. This section is concerned 
with obtaining a preliminary result that the configuration space $C(G-e, p)$ is compact. We will see that this is straightforward except for the special case of showing that $C(G, p)$ is bounded when $S=\mathcal{C}(1)$ i.e. $S$ is the cone defined by $x^{2}+y^{2}=z^{2}$. To solve this special case, we use a characterisation of realisations of $K_{n}$ in $\mathbb{R}^{m}$ which follows from the work of Cayley and Menger, see [3].

Let $V=V\left(K_{n}\right)=\left\{v_{1}, v_{2}, \ldots, v_{n}\right\}$ and $d$ be a map from $E\left(K_{n}\right)$ to the non-negative real numbers with $d\left(v_{i} v_{j}\right)=d_{i, j}$. The Cayley-Menger matrix $C M(V, d)=\left(c_{i, j}\right)$ is the symmetric $(n+1) \times(n+1)$-matrix in which $c_{i, i}=0$ for all $0 \leq i \leq n$, $c_{0, i}=1=c_{i, 0}$ for all $1 \leq i \leq n$, and $c_{i, j}=d_{i, j}$ for all distinct $1 \leq i, j \leq n$.

Theorem 8 Let $V=V\left(K_{n}\right)=\left\{v_{1}, v_{2}, \ldots, v_{n}\right\}$ and $d$ be a map from $E\left(K_{n}\right)$ to the non-negative real numbers with $d\left(v_{i} v_{j}\right)=d_{i, j}$. Then there exists a map $p: V \rightarrow \mathbb{R}^{t}$ with $\left\|p\left(v_{i}\right)-p\left(v_{j}\right)\right\|^{2}=d_{i, j}$ for all $1 \leq i<j \leq n$ if and only if $(-1)^{|U|} \operatorname{det} C M\left(U,\left.d\right|_{U}\right) \geq 0$ for all $U \subseteq V$ with $3 \leq|U| \leq t+1$ and $\operatorname{det} C M\left(U,\left.d\right|_{U}\right)=0$ for all $U \subseteq V$ with $|U|=t+2$.

Note that, if $p$ exists, then $-\frac{1}{4} \operatorname{det} C M\left(U,\left.d\right|_{U}\right)$ is the square of the area of the triangle induced by the points in $p(U)$ when $|U|=3$, and $\frac{1}{288} \operatorname{det} C M\left(U,\left.d\right|_{U}\right)$ is the square of the volume of the tetrahedron induced by the points in $p(U)$ when $|U|=4$. More generally, if $|U|=k+1$, then $(-1)^{k+1} \frac{1}{2^{k}(k !)^{2}} \operatorname{det} C M\left(U,\left.d\right|_{U}\right)$ is the square of the ' $k$-dimensional content' of the (possibly degenerate) simplex induced by the points in $p(U)$.

Lemma 15 Let $(G, p)$ be a generic realisation of a graph $G=(V, E)$ on the cone with rank $\left.d F_{G}\right|_{p}=3 n-2$. Then $C(G, p)$ is bounded.

Proof Let $V=\left\{v_{1}, v_{2}, \ldots, v_{n}\right\}$. If $G$ is not connected then Theorem 5 implies that $(G, p)$ is the disjoint union of two rigid frameworks and hence $C(G, p)$ is bounded. Thus we may assume that $G$ is connected. This implies that there exists a constant $K$ such that, for every equivalent framework $(G, q)$ on the cone, we have $\| q\left(v_{i}\right)-$ $q\left(v_{j}\right) \|^{2}<K$ for all $v_{i}, v_{j} \in V$.

Suppose $C(G, p)$ is not bounded. Then there exist a sequence of frameworks $\left(G, q_{1}\right),\left(G, q_{2}\right), \ldots$ on the cone such that $\left(G, q_{t}\right)$ is equivalent to $(G, p)$ and $\left\|q_{t}(v)\right\|^{2}>t$ for all $v \in V$. Let $d_{i, j, t}=\left\|q_{t}\left(v_{i}\right)-q_{t}\left(v_{j}\right)\right\|^{2}$. Then $d_{i, j, t}$ is a bounded sequence of real numbers for all $1 \leq i<j \leq n$ and hence we may choose a sequence $t_{1}, t_{2}, \ldots$ such that each subsequence $d_{i, j, t_{s}}$ converges. Let $d_{i, j}=\lim _{s \rightarrow \infty} d_{i, j, t_{s}}$ for all $1 \leq i<j \leq n$.

Let $K_{n}$ be the complete graph on $V$ and $d_{t}: E\left(K_{n}\right) \rightarrow \mathbb{R}$ by $d_{t}\left(v_{i} v_{j}\right)=d_{i, j, t}$ for all $t \geq 1$ and all $v_{i}, v_{j} \in V$. Then $\lim _{s \rightarrow \infty} d_{t_{s}}=d$ where $d: E\left(K_{n}\right) \rightarrow \mathbb{R}$ by $d\left(v_{i} v_{j}\right)=d_{i, j}$. Since $q_{t}: V \rightarrow \mathbb{R}^{3}$ we have $(-1)^{|U|} \operatorname{det} C M\left(U,\left.d_{t}\right|_{U}\right) \geq 0$ for all $U \subset V$ with $|U|=3,4$ by Theorem 8. Hence $(-1)^{|U|} \operatorname{det} C M\left(U,\left.d\right|_{U}\right)=$ $\lim _{s \rightarrow \infty}(-1)^{|U|} \operatorname{det} C M\left(U,\left.d_{t_{s}}\right|_{U}\right) \geq 0$ when $|U|=3$, 4. Furthermore, the facts that the maximum curvature at a point on $\mathcal{C}$ decreases to zero as the point moves away from the origin and $\left\|q\left(v_{i}\right)-q\left(v_{j}\right)\right\|^{2}<K$ for all $v_{i}, v_{j} \in V$ imply that the volume of the tetrahedron induced by $q_{t}(U)$ will converge to zero as $t \rightarrow \infty$. The remark immediately after Theorem 8 now implies that $\operatorname{det} C M\left(U,\left.d\right|_{U}\right)=$ $\lim _{s \rightarrow \infty} \operatorname{det} C M\left(U,\left.d_{t_{s}}\right|_{U}\right)=0$ for all $U \subset V$ with $|U|=4$. Theorem 8 now gives 
us a map $q: V \rightarrow \mathbb{R}^{2}$ such that $\left\|q\left(v_{i}\right)-q\left(v_{j}\right)\right\|^{2}=d_{i, j}$ for all $1 \leq i<j \leq n$. We can use the isometries of $\mathbb{R}^{2}$ to move $\left(K_{n}, q\right)$ so that the first three coordinates of $q$ are zero. Since each $d_{i, j}$ is a polynomial in the components of $q$, this implies that $\operatorname{td}[\mathbb{Q}(d): \mathbb{Q}] \leq 2 n-3$. On the other hand, Lemma 7 and the facts that $(G, p)$ is generic on the cone and rank $\left.d F_{G}\right|_{p}=3 n-2$ imply that $\operatorname{td}\left[\mathbb{Q}\left(d_{G}\right): \mathbb{Q}\right] \geq 2 n-2$, where $d_{G}=\left\{\left\|p\left(v_{i}\right)-p\left(v_{j}\right)\right\|^{2}: v_{i} v_{j} \in E\right\}$. This gives a contradiction since we have $d_{i, j}=d_{i, j, t}=\left\|p\left(v_{i}\right)-p\left(v_{j}\right)\right\|^{2}$ for all $t \geq 1$ and all $v_{i} v_{j} \in E$, and hence $\mathbb{Q}\left(d_{G}\right) \subseteq \mathbb{Q}(d)$.

Lemma 16 Let $(G, p)$ be generic framework on $S$ with rank $\left.d F_{G}\right|_{p}=3 n-(\ell+1)$. Then $C(G, p)$ is a compact subset of $\mathbb{R}^{3 n}$ and $q(v) \neq(0,0,0)$ for all $q \in C(G, p)$ and $v \in V$.

Proof We first show that $C(G, p)$ is closed. Let $p_{1}, p_{2}, \ldots$ be a sequence of points in $C(G, p)$ which converge to a limit point $p \in \mathbb{R}^{3 n}$. Then $\left(G, p_{1}\right),\left(G, p_{2}\right), \ldots$ is a sequence of frameworks in standard position on $S$ which are equivalent to $(G, p)$ and converge to a limit $(G, q)$. It is easy to see that $(G, q)$ will be in standard position on $S$ and equivalent to $(G, p)$. Hence $q \in C(G, p)$.

We next verify boundedness. When $S=\mathcal{E}$ or $\mathcal{S}$, this follows from the fact $C(G, p) \subset S$ and $S$ is bounded. When $S=\mathcal{Y}$ or $S=\mathcal{C}(r)$ with $r \neq\left(r_{1}, r_{1}, \ldots, r_{1}\right)$, it follows from the facts that $G$ is connected (by Theorem 4 ) and that $(G, q)$ is in standard position on $S$ and equivalent to $\left(G, p_{0}\right)$ for all $q \in C(G, p)$. Boundedness follows when $S=\mathcal{C}(1)$ by Lemma 15 .

It remains to show that $q(v) \neq(0,0,0)$ for all $q \in C(G, p)$ and $v \in V$. This is trivial when $S \neq \mathcal{C}$, so we can assume that $S=\mathcal{C}$. In this case Lemma 7 implies that $\operatorname{td}\left[\mathbb{Q}\left(r, f_{G}(q)\right): \mathbb{Q}(r)\right] \geq 2 n-2$ for all $q \in C(G, p)$ and hence $q(v) \neq(0,0,0)$ for all $v \in V$.

\section{Global Rigidity}

We can now show that the known necessary conditions for global rigidity in $\mathbb{R}^{d}$ given in Theorem 1 have natural analogues for generic frameworks on surfaces. First we consider $k$-connectivity.

Proposition 1 Let $(G, p)$ be a generic globally rigid framework on $S$ with at least four vertices. Then $G$ is $k$-connected where $k=3$ if $S=\mathcal{S}, k=2$ if $S \in\{\mathcal{Y}, \mathcal{C}\}$ and $k=1$ if $S=\mathcal{E}$.

Proof Assume $G$ is not $k$-connected. Then we have $G=G_{1} \cup G_{2}$ for subgraphs $G_{i}=\left(V_{i}, E_{i}\right)$ with $\left|V_{1} \cap V_{2}\right| \leq k-1$. Let $p_{1}=\left.p\right|_{V_{1}}$ and $p_{2}=\left.p\right|_{V_{2}}$. Let $(G, q)$ be obtained from $(G, p)$ by reflecting $\left(G_{2}, p_{2}\right)$ in a plane which contains $p\left(V_{1} \cap V_{2}\right)$ and also contains: the origin when $S=\mathcal{S}$; the $z$-axis when $S \in\{\mathcal{Y}, \mathcal{C}\}$; the $y$ - and $z$-axes when $S=\mathcal{E}$. Then $(G, q)$ is a framework on $S$ and is equivalent but not congruent to $(G, p)$.

We next consider redundant rigidity. 
Theorem 9 Suppose $\left(G, p_{0}\right)$ is quasi-generic and globally rigid on $S$ and $n \geq 7-\ell$. Then $\left(G, p_{0}\right)$ is redundantly rigid on $S$.

Proof Without loss of generality we may assume that $\left(G, p_{0}\right)$ is in standard position. Since $\left(G, p_{0}\right)$ is globally rigid, it is rigid. Suppose, for a contradiction, that $\left(G, p_{0}\right)$ is not redundantly rigid. Choose $e \in E$ such that $\left(G-e, p_{0}\right)$ is not rigid. Since $n \geq 7-\ell$, we have rank $d f_{[G]}(p)=2 n-\ell$ and rank $d f_{[G-e]}(p)=2 n-\ell-1$. Hence, by Lemma $12, C\left(G-e, p_{0}\right)=f_{[G-e]}^{-1}\left(f_{[G-e]}\left(p_{0}\right)\right)$ is a one dimensional submanifold of $\mathcal{M}$.

Let $\mathcal{O}$ be the component of $C\left(G-e, p_{0}\right)$ which contains $p_{0}$. Lemma 16 and the classification of one dimensional manifolds tells us that $\mathcal{O}$ is diffeomorphic to a circle. For any $q \in \mathcal{M}$, we consider the framework $(G, q)$ and define the map $f_{e}: \mathcal{M} \rightarrow \mathbb{R}$ by $f_{e}(q)=\|q(u)-q(v)\|^{2}$, where $e=u v$. Then $f_{[G]}=\left(f_{e}, f_{[G-e]}\right)$. Let $f_{[e]}=\left.f_{e}\right|_{C\left(G-e, p_{0}\right)}$.

By Lemma 11, $f_{[G-e]}\left(p_{0}\right)$ is a regular value of $f_{[G-e]}$. Since $C\left(G-e, p_{0}\right)=$ $f_{[G-e]}^{-1}\left(f_{[G-e]}\left(p_{0}\right)\right)$, Lemma 3 gives

$$
\left.\operatorname{rank} d f_{[e]}\right|_{p_{0}}=\left.\operatorname{rank} d f_{[G]}\right|_{p_{0}}-\left.\operatorname{rank} d f_{[G-e]}\right|_{p_{0}}=2 n-\ell-(2 n-(\ell+1))=1 .
$$

Hence $p_{0}$ is not a critical point of $f_{[e]}$, and there exists $q_{1}, q_{2} \in \mathcal{O}$ with $f_{e}\left(q_{1}\right)<$ $f_{e}\left(p_{0}\right)<f_{e}\left(q_{2}\right)$. There are two paths in $\mathcal{O}$ between $q_{1}$ and $q_{2}$, so by the Intermediate Value Theorem there exists a $p_{1} \in \mathcal{O}$ with $p_{1} \neq p_{0}$ and $f_{e}\left(p_{1}\right)=f_{e}\left(p_{0}\right)$. Then $\left(G, p_{0}\right)$ is equivalent to $\left(G, p_{1}\right)$. We may assign an orientation to $\mathcal{O}$ and suppose that $p_{1}$ has been chosen such that $p_{1}$ is as close to $p_{0}$ as possible when we traverse $\mathcal{O}$ in the forward direction. If $\left(G, p_{0}\right)$ is not congruent to $\left(G, p_{1}\right)$ we are done so we may assume that $\left(G, p_{0}\right)$ is congruent to $\left(G, p_{1}\right)$. Then Lemma 14 implies there is an isometry $\iota$ of $S$ such that $\iota\left(p_{0}\right)=p_{1}$. Since $\left(G, p_{0}\right)$ and $\left(G, p_{1}\right)$ are both in standard position with respect to $v_{1}, \iota$ is a discrete isometry of $S$; i.e. $\iota$ is a composition of reflections of $S$ in some planes. Given any point $p \in \mathcal{O}$, let $p^{-1}=\iota(p)$. Let $\alpha:[0,1] \rightarrow \mathcal{O}$ be a path in $\mathcal{O}$ from $p_{0}$ to $p_{1}$, and put $p_{t}=\alpha(t)$ for all $t \in[0,1]$. Define $\alpha^{-1}:[0,1] \rightarrow \mathcal{O}$ by $\alpha^{-1}(t)=p_{t}^{-1}$. Then $\alpha^{-1}$ is a path in $\mathcal{O}$ from $p_{1}$ to $p_{0}$.

First suppose that $\alpha$ and $\alpha^{-1}$ have different images in $\mathcal{O}$. Then $\alpha$ and $\alpha^{-1}$ cover $\mathcal{O}$. Without loss of generality suppose that $f_{e}$ increases as we pass through $p_{0}$ in the forward direction. Then $f_{e}$ also increases as we pass through $p_{1}$ in the forward direction. Hence there exists $t_{1}, t_{2}$ with $0<t_{1}<t_{2}<1$ such that $f_{e}\left(p_{t_{1}}\right)>f_{e}\left(p_{0}\right)$ and $f_{e}\left(p_{t_{2}}\right)<f_{e}\left(p_{1}\right)=f_{e}\left(p_{0}\right)$. By the Intermediate Value Theorem, there exists $t_{3} \in\left[t_{1}, t_{2}\right]$ with $f_{e}\left(p_{t_{3}}\right)=f_{e}\left(p_{0}\right)$. Then $\left(G, p_{t_{3}}\right)$ is equivalent to $\left(G, p_{0}\right)$ and $p_{t_{3}}$ contradicts the choice of $p_{1}$.

Now suppose $\alpha$ and $\alpha^{-1}$ have the same image in $\mathcal{O}$. Then $\alpha$ and $\alpha^{-1}$ traverse the same segment of $\mathcal{O}$ in opposite directions. Call the direction from $p_{0}$ to $p_{1}$ forward. By the Intermediate Value Theorem there exists $t \in[0,1]$ such that $\alpha(t)=\alpha^{-1}(t)$. Putting $\alpha(t)=p_{t}$ we have $p_{t}^{-1}=p_{t}$. We will show that this contradicts the fact that $\left(G, p_{0}\right)$ is quasi-generic. Consider the following cases.

Case 1: $S=\mathcal{S}$. Then $\iota$ is the unique reflection in the plane $x=0$. Thus, for any realisation $(G, p)$, if $p\left(v_{i}\right)=\left(x_{i}, y_{i}, z_{i}\right)$ we have $p^{-1}\left(v_{i}\right)=\left(-x_{i}, y_{i}, z_{i}\right)$. Since $p_{t}\left(v_{i}\right)=p_{t}^{-1}\left(v_{i}\right)$ we have $p_{t}\left(v_{i}\right)=\left(0, y_{i}, z_{i}\right)$ for all $v_{i} \in V$. Since $p_{t}\left(v_{1}\right)=$ $\left(0, y_{1}, 0\right)$ and $x_{i}^{2}+y_{i}^{2}+z_{i}^{2}=r_{i}$ for all $2 \leq i \leq n$ this gives $\operatorname{td}\left[\mathbb{Q}\left(r, p_{t}\right): \mathbb{Q}(r)\right] \leq$ 
$n-1$. But $f_{G-e}\left(p_{t}\right)=f_{G-e}\left(p_{0}\right)$ and $\operatorname{td}\left[\mathbb{Q}\left(r, f_{G}\left(p_{0}\right)\right): \mathbb{Q}(r)\right]=2 n-3$, hence $\operatorname{td}\left[\mathbb{Q}\left(r, f_{[G-e]}\left(p_{t}\right)\right): \mathbb{Q}(r)\right]=2 n-4$, which gives a contradiction.

Case 2: $S=\mathcal{Y}$. Then $\iota$ is a composition of reflections in the plane $z=0$ and the plane through $(0,1,0)$ and the $z$-axis.

We first consider the subcase where $\iota$ is the reflection in the $z=0$ plane. Then, for any realisation $(G, p)$, if $p\left(v_{i}\right)=\left(x_{i}, y_{i}, z_{i}\right)$ we have $p^{-1}\left(v_{i}\right)=\left(x_{i}, y_{i},-z_{i}\right)$. Since $p_{t}\left(v_{i}\right)=p_{t}^{-1}\left(v_{i}\right)$ we have $p_{t}\left(v_{i}\right)=\left(x_{i}, y_{i}, 0\right)$ for all $v_{i} \in V$. Since $p_{t}\left(v_{1}\right)=$ $\left(0, y_{1}, 0\right)$ and $x_{i}^{2}+y_{i}^{2}=r_{i}$ for all $2 \leq i \leq n$ this gives $\operatorname{td}\left[\mathbb{Q}\left(r, p_{t}\right): \mathbb{Q}(r)\right] \leq$ $n-1$. But $f_{G-e}\left(p_{t}\right)=f_{G-e}\left(p_{0}\right)$ and $\operatorname{td}\left[\mathbb{Q}\left(r, f_{G}\left(p_{0}\right)\right): \mathbb{Q}(r)\right]=2 n-2$, hence $\operatorname{td}\left[\mathbb{Q}\left(r, f_{[G-e]}\left(p_{t}\right)\right): \mathbb{Q}(r)\right]=2 n-3$, which gives a contradiction.

Now consider the subcase where $\iota$ is the reflection in the plane through $\left(0, y_{1}, 0\right)$ and the $z$-axis. Then, for any realisation $(G, p)$, if $p\left(v_{i}\right)=\left(x_{i}, y_{i}, z_{i}\right)$ we have $p^{-1}\left(v_{i}\right)=$ $\left(-x_{i}, y_{i}, z_{i}\right)$. Since $p_{t}\left(v_{i}\right)=p_{t}^{-1}\left(v_{i}\right)$ we have $p_{t}\left(v_{i}\right)=\left(0, y_{i}, z_{i}\right)$ for all $v_{i} \in V$. As before we have $\operatorname{td}\left[\mathbb{Q}\left(r, p_{t}\right): \mathbb{Q}(r)\right] \leq n-1$. But td $\left[\mathbb{Q}\left(r, f_{[G-e]}\left(p_{t}\right)\right): \mathbb{Q}(r)\right]=$ $2 n-3$, which gives a contradiction.

Finally consider the subcase where $\iota$ is the composition of the reflection in the $z=0$ plane and in the plane through $\left(0, y_{1}, 0\right)$ and the $z$-axis. Recall that reflections generate a group, in this case $\mathbb{Z}_{2} \times \mathbb{Z}_{2}$, so this is indeed the last case. Then, for any realisation $(G, p)$, if $p\left(v_{i}\right)=\left(x_{i}, y_{i}, z_{i}\right)$ we have $p^{-1}\left(v_{i}\right)=\left(-x_{i}, y_{i},-z_{i}\right)$. Since $p_{t}\left(v_{i}\right)=p_{t}^{-1}\left(v_{i}\right)$ we have $p_{t}\left(v_{i}\right)=\left(0, y_{i}, 0\right)$ for all $v_{i} \in V$. Since $x_{i}^{2}+y_{i}^{2}=r_{i}^{2}$ we have td $\left[\mathbb{Q}\left(r, p_{t}\right): \mathbb{Q}(r)\right]=0$. But $\operatorname{td}\left[\mathbb{Q}\left(r, f_{[G-e]}\left(p_{t}\right)\right): \mathbb{Q}(r)\right]=2 n-3$, which gives a contradiction.

Case 3: $S=\mathcal{C}$. Then $\iota$ is the reflection in the plane through $\left(0, y_{1}, z_{1}\right)$ and the $z$ axis. Then, for any realisation $(G, p)$, if $p\left(v_{i}\right)=\left(x_{i}, y_{i}, z_{i}\right)$ we have $p^{-1}\left(v_{i}\right)=$ $\left(-x_{i}, y_{i}, z_{i}\right)$. Since $p_{t}\left(v_{i}\right)=p_{t}^{-1}\left(v_{i}\right)$ we have $p_{t}\left(v_{i}\right)=\left(0, y_{i}, z_{i}\right)$ for all $v_{i} \in V$. Hence td $\left[\mathbb{Q}\left(r, p_{t}\right): \mathbb{Q}(r)\right] \leq n-1$. But td $\left[\mathbb{Q}\left(r, f_{[G-e]}\left(p_{t}\right)\right): \mathbb{Q}(r)\right]=2 n-2$, which gives a contradiction.

Case 4: $S=\mathcal{E}$. Then $\iota$ is a composition of reflections in the plane $x=0$, the plane $y=0$ and the plane $z=0$. First consider the subcase when $\iota$ is the reflection in the plane $x=0$. Then, for any realisation $(G, p)$, if $p\left(v_{i}\right)=\left(x_{i}, y_{i}, z_{i}\right)$, we have $p^{-1}\left(v_{i}\right)=\left(-x_{i}, y_{i}, z_{i}\right)$. Since $p_{t}\left(v_{i}\right)=p_{t}^{-1}\left(v_{i}\right)$ we have $p_{t}\left(v_{i}\right)=\left(0, y_{i}, z_{i}\right)$ for all $v_{i} \in V$. Hence td $\left[\mathbb{Q}\left(p_{t}\right): \mathbb{Q}\right] \leq n$. But td $\left[\mathbb{Q}\left(r, f_{[G-e]}\left(p_{t}\right)\right): \mathbb{Q}(r)\right]=2 n-1$, which gives a contradiction. The other subcases follow similarly.

The necessary conditions for global rigidity given in Proposition 1 and Theorem 9 are independent since, for each $S \in\{\mathcal{S}, \mathcal{Y}, \mathcal{C}, \mathcal{E}\}$, there are examples of generic frameworks $(G, p)$ on $S$ such that $G$ is $k$-connected for $k$ as in Proposition 1, but $(G, p)$ is not redundantly rigid, and examples such that $(G, p)$ is redundantly rigid but not $k$-connected.

\section{Concluding Remarks}

1. Theorem 2 and the result of Connelly and Whiteley [5] that generic global rigidity in $\mathbb{R}^{2}$ and the sphere are equivalent, show that the necessary conditions for generic global rigidity given in Proposition 1 and Theorem 9 are sufficient for the sphere. 
We conjecture that they are also sufficient for (concentric families of) cylinders and cones.

Conjecture 1 Let $(G, p)$ be a generic framework on $S$ where $S \in\{\mathcal{Y}, \mathcal{C}\}$. Then $(G, p)$ is globally rigid on $S$ if and only if either $G$ is a complete graph on at most four vertices or $G$ is 2-connected and $(G, p)$ is redundantly rigid.

There is some hope that the special case of Conjecture 1 when $S=\mathcal{Y}(r)$ and $\operatorname{td}[\mathbb{Q}(r): \mathbb{Q}]=n$ will be resolved in the near future. The third author gives a recursive construction for 2-connected graphs with $2 n-1$ edges which are redundantly rigid on $\mathcal{Y}$ in [14], and we are currently extending this construction to all 2-connected redundantly rigid graphs. In addition, the first and third authors [10] have shown that the main operation used in these recursive constructions, the so called Henneberg type 2 operation, preserves generic global rigidity on $\mathcal{Y}(r)$ when $\operatorname{td}[\mathbb{Q}(r): \mathbb{Q}]=n$.

We know of no examples for which the necessary conditions given in Proposition 1 and Theorem 9 fail to be sufficient to imply generic global rigidity on the ellipsoid. On the other hand we do not even know how to characterise generic rigidity for the ellipsoid.

2. If true, Conjecture 1 would give a polynomial algorithm to check generic global rigidity on the cylinder and cone: redundant rigidity can be checked in polynomial time, see for example [2] or [12], as can 2-connectivity, see [18].

3. The methods in this paper can be adapted to prove analogues of Theorem 9 for a number of other surfaces including tori, elliptical cylinders and elliptical cones. It is conceivable that the methods will apply to any irreducible 2-dimensional algebraic variety embedded in $\mathbb{R}^{3}$.

Acknowledgments We would like to thank the School of Mathematics, University of Bristol for supporting the first author's visits during which most of this research took place. We also thank Edward Crane for helpful conversations concerning generic points on algebraic varieties and the referees for their helpful comments and suggestions.

\section{References}

1. Anderson, B.D., Belhumeur, P.N., Eren, T., Goldenberg, D.K., Morse, A.S., Whiteley, W., Yang, Y.R.: Graphical properties of easily localizable sensor networks. Wirel. Netw. 15, 177-191 (2009)

2. Berg, A. R., Jordán, T.: Algorithms for graph rigidity and scene analysis. In: Di Battista, G., Zwick, U. (eds.) Proceedings of the 11th Annual European Symposium on Algorithms (ESA). Lecture Notes in Computer Science, vol. 2832, pp. 78-89. Springer, Berlin (2003)

3. Blumenthal, L.M.: Theory and Applications of Distance Geometry, vol. 15. Chelsea, Bronx (1970)

4. Connelly, R.: Generic global rigidity. Discrete Comput. Geom. 33, 549-563 (2005)

5. Connelly, R., Whiteley, W.J.: Global rigidity: the effect of coning. Discrete Comput. Geom. 43, 717-735 (2010)

6. Hartshorne, R.: Algebraic Geometry. Springer, New York (1977)

7. Hendrickson, B.: Conditions for unique graph realizations. SIAM J. Comput. 21, 65-84 (1992)

8. Jackson, B., Jordán, T.: Connected rigidity matroids and unique realizations of graphs. J. Comb. Theory Ser. B 94, 1-29 (2005)

9. Jackson, B., Keevash, P.: Necessary conditions for the global rigidity of direction-length frameworks. Discrete Comput. Geom. 46, 72-85 (2011)

10. Jackson, B., Nixon, A.: Stress matrices and global rigidity of frameworks on surfaces. http://arxiv.org/ abs/1406.5996, submitted (2014) 
11. Laman, G.: On graphs and rigidity of plane skeletal structures. J. Eng. Math. 4, 331-340 (1970)

12. Lee, A., Streinu, I.: Pebble game algorithms and sparse graphs. Discrete Math. 308, 1425-1437 (2008)

13. Milnor, J.W.: Topology from the Differentiable Viewpoint. The University Press of Virginia, Charlottesville (1965)

14. Nixon, A.: A constructive characterisation of circuits in the simple (2,2)-sparsity matroid. European J. Combin. 42, 92-106 (2014)

15. Nixon, A., Owen, J.C., Power, S.C.: Rigidity of frameworks supported on surfaces. SIAM J. Discrete Math. 26, 1733-1757 (2012)

16. Nixon, A., Owen, J. C., Power, S. C.: A characterisation of generically rigid frameworks on surfaces of revolution. SIAM J. Discrete Math. (to appear)

17. Seidenberg, A.: A new decision method for elementary algebra. Ann. Math. 2(60), 365-374 (1954)

18. Tarjan, R.: Depth-first search and linear graph algorithms. SIAM J. Comput. 1, 146-160 (1972)

19. Tarski, A.: A Decision Method for Elementary Algebra and Geometry. RAND Corporation, Santa Monica (1948)

20. Zelazo, D., Franchi, A., Allgower, F., Bulthoff, H., Giordano, P.R.: Rigidity maintenance control for multi-robot systems. In: Proceedings of Robotics: Science and Systems, Sydney, Australia (2012) 